\title{
The Use of the Alcohol Use Disorders Identification Test - Consumption as an Indicator of Hazardous Alcohol Use among University Students
}

\author{
Sanne Verhoog ${ }^{\text {a, b }}$ Jolien M. Dopmeijer ${ }^{c, g} \quad$ Jannet M. de Jonge ${ }^{c}$ \\ Claudia M. van der Heijde ${ }^{d}$ Peter Vonk ${ }^{d}$ Rob H.L.M. Bovens ${ }^{e}$ Michiel R. de Boer ${ }^{f}$ \\ Trynke Hoekstra $^{f}$ Anton E. Kunst ${ }^{a}$ Reinout W. Wiers ${ }^{g}$ Mirte A.G. Kuipers ${ }^{a}$ \\ ${ }^{a}$ Academic Medical Center, Department of Public Health, University of Amsterdam, Amsterdam, The Netherlands; ${ }^{b}$ Institute \\ of Social and Preventive Medicine, University of Bern, Bern, Switzerland; ' ${ }^{C}$ Department of Health and Welfare, Windesheim \\ University of Applied Sciences, Research Group Mental Healthcare and Society, Zwolle, The Netherlands; ${ }^{\mathrm{d}}$ Department of \\ Research, Student Health Service, Development and Prevention, University of Amsterdam, Amsterdam, The Netherlands; \\ eTranzo, Scientific Center for Care and Welfare, University of Tilburg, Tilburg, The Netherlands; ${ }^{\mathrm{f}}$ Department of Health \\ Sciences, VU University, Section Methodology and Applied Statistics, Amsterdam, The Netherlands; ${ }^{9}$ Department of \\ Psychology, Addiction Development and Psychopathology Lab, University of Amsterdam, Amsterdam, The Netherlands
}

\section{Keywords}

Alcohol use screening · Hazardous alcohol use ·

University students · Concurrent validity · Alcohol use disorders identification test-consumption

\begin{abstract}
Background: Hazardous drinking among students in higher education is a growing concern. The alcohol use disorders identification test (AUDIT) is the gold standard screening instrument for hazardous drinking in the adult population, for which an abbreviated version has been developed: the AUDIT-Consumption (AUDIT-C). Currently, there's no gold standard for identifying hazardous drinking among students in higher education and little evidence regarding the concurrent validity of the AUDIT-C as a screening instrument for this group. This study investigated the concurrent validity of the AUDIT-C in a sample of university students and suggests the most appropriate cutoff points. Methods: Cross-sectional data of health surveys from 5,401 university and university of applied sciences in the Netherlands were used. Receiver operating characteristic (ROC) curves, sensitivity, specificity,
\end{abstract}

and positive and negative predictive values for different cutoff scores of AUDIT-C were calculated for the total sample and for subgroups stratified by age, gender, and educational level. AUDIT-score $\geq 11$ was used as the criterion of hazardous and harmful drinking. Results: Twenty percent of students were hazardous and harmful drinkers. The area under the ROC curve was 0.922 (95\% Cl 0.914-0.930). At an AUDIT-C cutoff score of $\geq 7$, sensitivity and specificity were both $>80 \%$, while other cutoffs showed less balanced results. A cutoff of $\geq 8$ performed better among males, but for other subgroups $\geq 7$ was most suitable. Conclusion: AUDIT-C seems valid in identifying hazardous and harmful drinking students, with suggested optimal cutoffs 7 (females) or 8 (males). However, considerations regarding avoiding false-positives versus false-negatives, in relation to the type of intervention following screening, could lead to selecting different cutoffs.

(C) 2019 The Author(s)

Published by S. Karger AG, Basel

S.V. and J.M.D. shared first authorship. R.W.W. and M.A.G.K. shared last authorship.

\begin{tabular}{ll}
\hline KARGER & $\begin{array}{l}\text { Karger } \\
\text { Published The Author(s) }\end{array}$ \\
& $\begin{array}{l}\text { Kargerger AG, Basel } \\
\text { Open ciccess }\end{array}$ \\
E-Mail karger@karger.com & This article is licensed under the Creative Commons Attribution- \\
wwww.karger.com/ear & $\begin{array}{l}\text { NonCommercial-NoDerivatives 4.0 International License (CC BY- } \\
\text { NC-ND) (http://www.karger.com/Services/OpenAccessLicense). } \\
\text { Usage and distribution for commercial purposes as well as any dis- } \\
\text { tribution of modified material requires written permission. }\end{array}$
\end{tabular}




\section{Introduction}

Students drink more than their peers who are not attending higher education [1-3], and alcohol use is the leading cause of injury and death among students $[4,5]$. Especially binge drinking (drinking 5 or more drinks in one occasion) is a highly prevalent risk behavior [6] that increases students' short-term risk of poor academic performance [7] and college drop-out [8] and their longterm risk of alcohol dependence and learning and memory impairments [7-10].

In Europe, the continent with the highest per capita alcohol consumption, hazardous alcohol use is very prevalent among students $[11,12]$. In the Netherlands (where this study took place), 24\% of students between 18 and 24 are hazardous drinkers. Hazardous drinking is defined as men consuming 6 or more and women 4 or more glasses of alcohol at least once a week [13]. This is much higher than in the general adult population, where $10 \%$ are hazardous drinkers [14].

Due to the societal acceptance of high levels of alcohol use as part of student culture, hazardous student drinking is often downplayed $[14,15]$. This might partly be driven by the idea that many students show natural recovery of hazardous drinking after a typical peak of drinking at a younger age, often without specific treatment [16-19]. However, because hazardous drinking is associated with short-term risk of poor academic performance, college drop-out, and long-term risk of alcohol use disorder (AUD), there is a need to identify hazardously drinking students in order to refer them to primarily, further alcohol assessments and secondarily, if needed, appropriate interventions. The societal acceptance on students' drinking behaviors impedes the identification of hazardous drinking students especially those being at risk of AUD and furthermore and most important, current screening instruments and cutoffs seem to do so too. These instruments cause an overestimation of students who seem to be at risk for AUD, which is probably the result of the high prevalence of binge drinking among students, based on which they quickly exceed the cutoff of hazardous drinking. Even though binge drinking is risky behavior that we want to identify, it is not enough to base hazardous drinking and being at risk for AUD on. However, there is no gold standard for a valid screening of hazardous alcohol use among students in higher education. Having an assessment instrument to identify these students by measuring prevalence and patterns of risk enables researchers, practitioners, and policymakers [20] to appropriately refer students to further alcohol assessments and interventions.
Cutoffs of screening instruments for hazardous drinking and being at risk for AUD are derived from the general adult population where hazardous drinking is less frequent than in the student population. Therefore, these screening instruments may identify a higher percentage of hazardously drinking students who are at risk for AUD, than are actually at risk and may need help. This would suggest that cutoffs should be higher in the student population than in the adult population, but there is a lack of information on which cutoff point would most accurately identify students with hazardous drinking behaviors, at risk for AUD.

The 10-item AUD identification test (AUDIT) [21] has been developed by the World Health Organization to identify people with hazardous drinking behaviors and AUDs [22] and is regarded as the gold standard questionnaire for screening hazardous and harmful drinking in mainly clinical settings for the adult population. The first 3 questions of the AUDIT, that is, the AUDIT-Consumption (AUDIT-C), measure the amount and frequency of drinking $[22,23]$. The second part assesses the frequency of experienced mental and physical problems due to alcohol consumption. According to studies in adults, the AUDIT-C is almost equally accurate in detecting hazardous drinking patterns and being at risk for AUD as the full AUDIT [23, 24]. In adults, a score of 4 for men and 3 for women on the AUDIT-C is considered optimal for identifying hazardous drinking or active AUDs [22] with sensitivity and specificity in the mid-90s and 80s, respectively. Important advantages of using the AUDIT-C instead of the full AUDIT are that the questionnaire is shorter, and the questions are less intrusive. The AUDIT-C may therefore have a lower risk of response bias and reporting bias.

The concurrent validity of the AUDIT-C among students has been evaluated in a limited number of studies, with different outcome measures. In a US study among 18-20 year olds, of which $89 \%$ were college students, the AUDIT-C performed best with cutoff scores of 6 for men and 5 for women [25] in the prediction of AUD (using DSM-IV criteria), with sensitivity and specificity between 68 and 78\%. Another study among US students concluded that a cutoff score of 7 should be used for men and 5 for women in order to detect at-risk drinking (defined by the number of drinks in a typical week and/or the total number of heavy drinking episodes in the past month) [26]. To our knowledge, no such studies, besides the use of the AUDIT-C in the detection of binge drinking [27], were performed in Europe. Results in Europe could be different, due to differences in legislative and 
cultural context, such as the minimum age of alcohol sales and the standard serving size of alcoholic beverages [28-29].

Given the diversity of student populations, the sensitivity and specificity of screening methods for hazardous alcohol use may not be the same across different subgroups. This diversity may go beyond gender differences. Because of the typical peak in drinking in a younger age [18-19], we will compare different age groups. Furthermore, university is more known for their drinking culture than a university of applied sciences. Therefore, we will also compare groups based on educational level.

We assume that the AUDIT-C provides us with a good screening method for hazardous alcohol use, with higher optimal cutoffs for students than for the general adult population. This may apply to an even larger extent for those subgroups of students that are more frequent drinkers (i.e., men, university students, and older students). The aim of this study is to examine the concurrent validity of the AUDIT-C and to examine whether the AUDIT-C is a valid screening instrument for hazardous drinking and being at risk for AUD among university students. We therefore examined the sensitivity and specificity of different AUDIT-C cutoff points for hazardous alcohol use, defined with the full AUDIT score. Additionally, we examined the validity for different subgroups of age, gender, and educational level (university and university of applied sciences).

\section{Materials and Methods}

Data Collection and Study Sample

Cross-sectional data were used from the February 2015 to May 2016 Student Health Check survey, carried out in a university and a university of applied sciences in Amsterdam [30]. The students were invited for the survey by student advisors and course managers through emails, newsletters, and TV screens on campus. Furthermore, a website specifically developed for this purpose, the www.studenthealthcheck.nl [30] with the self-monitor online is available throughout the whole school year. In addition, cross-sectional data from the December 2012 to January 2013 Study environment, Health and Study success survey, carried out in a university of applied sciences in Zwolle, were used. Students were invited by email.

Both surveys were aimed at improving students' recognition of their health problems at an early stage through self-monitoring and to provide personalized feedback [30]. Participants were extensively informed upfront about the objective and procedure. The participants provided written consent for the use of data.

Not all students who were invited completed the survey. For the Student Health Check in Amsterdam $(n=5,169)$, the response rate

The AUDIT-C as an Indicator of

Hazardous Alcohol Use is unknown, as there are multiple recruitment methods that could not be monitored. Only the response rate for the Study environment, Health and Study success in Zwolle could be calculated which amounted to $14.7 \%(n=2,332)$. A total of 7,501 students completed the questionnaire.

Nondrinkers were defined as those who indicated to never consume alcoholic beverages and were removed from the sample ( $n=985$; including 77 who had missing values). From the remaining 6,516 students, participants with one or more missing values on AUDIT items $(n=136)$ and for age, gender, and/or educational level $(n=54)$ were also excluded. Lastly, 925 participants were excluded because their age exceeded the target age range (17-25). The final sample included 5,401 respondents.

\section{Measures}

Alcohol use was measured with the AUDIT-C (Audit questions 1-3) [23]. The questions assessed frequency of drinking, typical number of drinks consumed on a drinking day, and frequency of binge drinking. Responses to each item were scored from 0 to 4 . The other 7 AUDIT questions were also asked, to generate the full AUDIT-score [19]. Because of the lack of a gold standard for screening hazardous alcohol use for students, hazardous drinking was measured with reaching or exceeding the recommended (full) AUDIT cutoff of 11 for students by Fleming et al. [31], for adequate sensitivity. Note that this score is higher than for the general population.

Respondents provided demographics including age, gender, and educational level. Educational level discriminated students in university from students in the University of Applied Sciences. Age was classified into 2 groups: $17-21$ and 22-25, based on the phase in their education (bachelor vs. master). In the Netherlands, students start their bachelor at age 17 or 18, which last for 3 or 4 years. After completion of the bachelor, most students continue with a master program of 1 or 2 years.

\section{Statistical Analysis}

All statistical analyses were performed using SPSS, version 24 [32]. First, sociodemographic characteristics, prevalence of hazardous drinking, and mean AUDIT-C scores were described for the total sample and by gender, age, and educational level. Next, sensitivity, specificity, positive predictive value (PPV), and negative predictive value (NPV) were calculated for each AUDIT-C cutoff score for the total sample and for the different subgroups. Sensitivity identified the true positive proportion and specificity the true negative proportion. PPV determined the proportion of students who actually were hazardous drinkers according to the AUDIT out of all students with a score above the AUDIT-C cutoff point (i.e., positive test). NPV determined the proportion of students who actually were nonproblematic drinkers according to the AUDIT out of all students with a score below the AUDIT-C cutoff point (i.e., negative test). Receiver operating characteristic (ROC) curve analysis was used to determine the area under the curve (AUC) [33], which represents the accuracy of the test. AUCs higher than 0.80 are generally considered sufficient [34]. The AUC was measured for the total study sample and for the different subgroups. Finally, a sensitivity analysis was undertaken in which we varied the AUDIT cutoff criterion to define hazardous alcohol use to 10 and 12 and compared results with the main analysis. 
Table 1. Characteristics of participants in number (\%) of participants unless otherwise is indicated

\begin{tabular}{lcrr}
\hline & Total sample & Hazardous drinkers* & AUDIT-C score, mean (SD) \\
\hline Total & $5,401(100)$ & $1,080(20)$ & $5.15(2.47)$ \\
Men & $1,833(33.9)$ & $588(32.1)$ & $6.17(2.53)$ \\
Women & $3,568(66.1)$ & $492(13.8)$ & $4.62(2.26)$ \\
Age, years 17-21 & $2,240(41.5)$ & $394(17.6)$ & $5.00(2.42)$ \\
Age, years 22-25 & $3,161(58.5)$ & $686(21.7)$ & $5.25(2.50)$ \\
University of applied sciences students & $3,688(68.3)$ & $613(16.6)$ & $4.94(2.50)$ \\
University students & $1,713(31.7)$ & $467(27.3)$ & $5.59(2.33)$ \\
\hline
\end{tabular}

* Percentage of total sample and subgroups, defined AUDIT $\geq 11$.

AUDIT-C, Alcohol Use Disorders Identification Test - Consumption.

\section{Results}

Table 1 presents hazardous drinking rates and AUDIT-C scores in the total sample and in subgroups. The mean score on the AUDIT-C for all students was 5.15 (SD 2.47). Men had the highest mean AUDIT-C score of all subgroups (6.17, SD 2.53), followed by university students (5.59, SD 2.33) and older students (5.25, SD 2.50). A total of 1,080 students $(20.0 \%)$ were identified as hazardous drinkers, based on the AUDIT. The prevalence of hazardous drinking across subgroups shows almost the same pattern as for mean AUDIT-C scores, with relatively high scores among males (32.1\%) and students aged $22-25$ years $(21.7 \%)$.

The distribution of participants with positive or negative test results on scores of the AUDIT-C was calculated. Cutoff points 3-6 had more participants who scored above (range 99.9-95.5\%) than under the cutoff point (range 0.1-4.5\%). For cutoff points 7 and 8, more participants scored under the cutoff point (12.2 and 30.1\%) than above ( 87.8 and $69.9 \%$ ). The proportion of hazardous drinkers was substantially higher among those above the AUDIT-C cutoff point than those under the cutoff point, except for cutoff point 9 . From cutoff point 9 onwards the distribution reversed.

Sensitivity, specificity, PPV, and NPV for the total sample for possible cutoff scores of the AUDIT-C are presented in Table 2. The AUC value for the ROC curve was 0.922 (95\% CI 0.914-0.930), representing a high probability that a hazardous drinker has a higher AUDIT-C score than a nonhazardous drinker. Sensitivity was high ( $>85$ ) for cutoff points 3-7, but declined rapidly for cutoff points $8(69.2)$ and 9 (39.9). Specificity was low $(<65)$ for cutoff points $3-6$, and only reached high values $(>85)$ at cutoff points 8 and 9 . At cutoff point 7 , specificity was
Table 2. Sensitivity, specificity, PPV, and NPV for different cutoff scores AUDIT-C for the total sample $(n=5,401)$

\begin{tabular}{lllll}
\hline $\begin{array}{l}\text { Cutoff score } \\
\text { AUDIT-C }\end{array}$ & Sensitivity & Specificity & PPV & NPV \\
\hline 3 & 99.9 & 20.8 & 24.0 & 99.0 \\
4 & 99.6 & 35.0 & 27.7 & 99.7 \\
5 & 98.4 & 52.5 & 34.1 & 99.3 \\
6 & 95.5 & 68.9 & 43.4 & 98.4 \\
7 & 87.8 & 83.4 & 56.9 & 96.5 \\
8 & 69.2 & 92.9 & 70.8 & 92.3 \\
9 & 39.9 & 97.9 & 82.3 & 86.6 \\
\hline
\end{tabular}

PPV, positive predictive value; NPV, negative predictive value; AUDIT-C, Alcohol Use Disorders Identification Test - Consumption.

moderate (83.4\%). For cutoff points 3-6 PPV was low $(<55)$, at cutoff point $7 \mathrm{PPV}$ was moderate, and for cutoff points 8 and 9 PPV was high. NPV was high for all cutoff points ( $>85)$. Overall, a cutoff of 7 showed the most balanced combination of sensitivity and specificity (i.e., for which sensitivity and specificity were acceptably high).

Table 3 shows the sensitivity, specificity, PPV, and NPV of different cutoff points of the AUDIT-C, stratified by gender, age, and educational level. The AUC was high for all subgroups (>0.9), indicating a good performance of the AUDIT-C. Sensitivity, specificity, PPV, and NPV showed the same pattern for the subgroups as for the total sample. However, sensitivity decreased more rapidly in women than in men, whereas specificity increased more rapidly. Students aged 22-25 and university students had a higher PPV than students aged 17-21 and higher vocational students, respectively. Results suggest a different optimal cutoff point for men (8) than for women (7), 
Table 3. Sensitivity, specificity, PPV, and NPV in percentages for different cutoff scores AUDIT-C stratified by gender, age, and educational level

\begin{tabular}{|c|c|c|c|c|c|c|c|}
\hline & \multicolumn{7}{|c|}{ Cutoff scores AUDIT-C } \\
\hline & 3 & 4 & 5 & 6 & 7 & 8 & 9 \\
\hline \multicolumn{8}{|l|}{$\operatorname{Men}(n=1,833)$} \\
\hline Sensitivity & 100.0 & 100.0 & 98.8 & 97.1 & 92.5 & 80.8 & 51.0 \\
\hline Specificity & 13.3 & 23.8 & 41.0 & 57.0 & 74.9 & 86.1 & 94.5 \\
\hline PPV & 35.3 & 38.3 & 44.1 & 51.6 & 63.6 & 73.3 & 81.5 \\
\hline Sensitivity & 99.8 & 99.2 & 98.0 & 93.5 & 82.1 & 55.3 & 25.2 \\
\hline Specificity & 23.8 & 39.6 & 57.2 & 73.7 & 86.8 & 95.6 & 99.3 \\
\hline PPV & 17.3 & 20.8 & 26.8 & 36.3 & 49.8 & 66.8 & 84.4 \\
\hline NPV & 99.9 & 99.7 & 99.4 & 98.6 & 96.8 & 93.0 & 89.2 \\
\hline \multicolumn{8}{|c|}{ Age, years $17-21(n=2,240)$} \\
\hline Sensitivity & 100.0 & 100.0 & 98.7 & 96.7 & 87.3 & 68.3 & 36.3 \\
\hline Sensitivity & 99.9 & 99.4 & 98.3 & 94.8 & 88.0 & 69.7 & 41.0 \\
\hline Specificity & 19.9 & 34.5 & 52.0 & 67.8 & 82.8 & 92.8 & 98.0 \\
\hline PPV & 21.7 & 29.6 & 36.2 & 44.9 & 58.7 & 73.0 & 84.9 \\
\hline NPV & 99.8 & 99.5 & 99.1 & 97.9 & 96.2 & 91.7 & 85.7 \\
\hline \multicolumn{8}{|c|}{ University of applied sciences students $(n=3,688)$} \\
\hline Sensitivity & 100.0 & 99.7 & 98.4 & 95.3 & 88.6 & 71.5 & 45.7 \\
\hline Specificity & 23.1 & 37.7 & 55.6 & 71.3 & 84.2 & 92.7 & 97.4 \\
\hline PPV & 20.6 & 24.2 & 30.6 & 39.8 & 52.8 & 66.0 & 77.8 \\
\hline NPV & 100.0 & 99.8 & 99.4 & 98.7 & 97.4 & 94.2 & 90.0 \\
\hline \multicolumn{8}{|c|}{ University students $(n=1,713)$} \\
\hline Sensitivity & 99.8 & 99.6 & 98.5 & 95.7 & 86.7 & 66.2 & 30.8 \\
\hline Specificity & 15.2 & 28.5 & 44.9 & 63.1 & 81.2 & 93.4 & 99.1 \\
\hline PPV & 30.6 & 34.3 & 40.1 & 49.3 & 63.4 & 79.0 & 92.9 \\
\hline
\end{tabular}

AUC (95\% CI) for men: 0.908 (0.895-0.922).

AUC (95\% CI) for women: $0.918(0.906-0.929)$.

AUC (95\% CI) for age 17-21: $0.924(0.912-0.937)$.

AUC (95\% CI) for age 22-25: $0.920(0.910-0.931)$

AUC (95\% CI) for higher vocational students: $0.928(0.919-0.938)$.

AUC (95\% CI) for University students: 0.912 (0.897-0.926).

PPV, positive predictive value; NPV, negative predictive value; AUDIT-C, Alcohol Use Disorders Identification Test - Consumption; AUC, area under the curve.

when sensitivity and specificity are most balanced. For various age and educational level subgroups, 7 seems the optimal cutoff point.

In a sensitivity analysis as shown in Table 4, the analysis was repeated with hazardous drinking defined by 2 alternative cutoff scores for the AUDIT; 10 and 12, respectively. The values of sensitivity, specificity, and NPV slightly changed, with a maximum change of $5 \%$. The PPV changed more, with a maximum of $10 \%$. This is due to the change of the prevalence of hazardous drinkers at different cutoff scores of the AUDIT.

The AUDIT-C as an Indicator of Hazardous Alcohol Use

\section{Discussion/Conclusion}

\section{Key Results}

The ROC results imply that the AUDIT-C is a valid test to identify hazardous drinking in the student population, as defined by the full AUDIT. Sensitivity and specificity outcomes were in balance and simultaneously high $(>80 \%)$ at cutoff point 7 for the total sample. The PPV was low $(<50 \%)$ for cutoff points 3-6 and increased with cutoff points $7-9$. The NPV was high $(>85 \%)$ for all cutoff points. These patterns were similar for groups of different 
Table 4. Sensitivity analysis $(n=5,401)$

\begin{tabular}{llllll}
\hline Cutoff point AUDIT-C & Cutoff point AUDIT & Sensitivity & Specificity & PPV & NPV \\
\hline 6 & 10 & 93.7 & 72.9 & 53.9 & 97.2 \\
& 11 & 95.5 & 68.9 & 43.4 & 98.4 \\
& 12 & 96.4 & 66.0 & 34.9 & 99.0 \\
\hline 7 & 10 & 84.0 & 87.1 & 68.7 & 94.1 \\
& 11 & 87.8 & 83.4 & 56.9 & 96.5 \\
& 12 & 90.5 & 80.4 & 46.7 & 97.8 \\
\hline 8 & 10 & 63.6 & 95.4 & 82.3 & 88.6 \\
& 11 & 69.2 & 92.9 & 70.8 & 92.3 \\
\hline
\end{tabular}

AUDIT, alcohol use disorders identification test; AUDIT-C, Alcohol Use Disorders Identification Test - Consumption; PPV, positive predictive value; NPV, negative predictive value.

ages and educational levels, but not for gender. The most balanced cutoff point was higher in males (8) compared to females (7).

\section{Comparison with Previous Studies}

Our findings are largely in agreement with those observed in 2 US studies, examining the use of the AUDIT$\mathrm{C}$ in a student population $[25,26]$. These 2 studies found AUCs of 0.83 and 0.89 , respectively, which is comparable with our findings. Their recommended cutoff scores of, respectively, 5 and 6 are lower than our most balanced combination of sensitivity and specificity at cutoff 7 . Differences in cutoff scores might be due to standard drinks being smaller in Europe (10 g in the Netherlands) compared to the United States (14 g). Furthermore, lower cutoff scores in the United States might be explained by other legislation in Europe compared to the US with regard to the age limit of alcohol consumption.

In addition, Kelly et al. [25] also found a higher AUC for women than for men, and a higher cutoff score for men (6) was recommended compared to women (5). DeMartini and Carey [26] also proposed a higher cutoff score in men (7) than for women (5).

Another study examining the validity of the AUDIT-C for at-risk drinking among students recommended a cutoff score of 5 for women and 7 for males [35], whereby at-risk drinking was defined as 14 or more drinks per week for males and 7 or more drinks per week for females. This is slightly lower than the recommended cutoff scores in our study, which could again be due to other legislation in the United States compared to Europe.

A study conducted in Sweden [36] examined the ability of the AUDIT-C to discriminate between a group of problem drinkers and nonproblem drinkers, whereby problem drinking was defined as a treatment-seeking population and the general population comprised the nonproblem drinkers. They found an optimal cutoff point of 6 with an AUC of 0.60 and 0.32 and 0.92 sensitivity and specificity, respectively. The optimal cutoff point and AUC are lower than in our study, which might be due to the difference in definition of problem drinking between both studies.

A review reported that the AUDIT-C performs almost equally well as the full AUDIT in predicting alcohol use problems and AUD [37]. This review also recommended separate cutoffs for men and women when using the AUDIT-C. The recommended cutoff score for detecting hazardous drinking is 4 for men and 3 for women. However, of the 15 studies examined in the review, none used a college sample, and hazardous drinking was defined in various ways. Furthermore, most studies were conducted among primary care patients or participants with mood or anxiety disorders. Therefore, the findings of this review are not comparable to the findings in our study.

\section{Implications}

The results showed that the AUDIT-C cutoff score of 4 (proposed for the general population by Saunders et al. [22]) will lead to many false-positives in (Dutch or European) university students. Based on our findings, we recommend cutoffs of 8 for male and 7 for female students. The AUDIT-C is intended to determine eligibility of students for further alcohol assessments, but if needed, students can be referred to targeted interventions as a result of these assessments.

Regarding interventions, the choice of the cutoff points depends on the country (and related size of standard 
drinks) and the need to avoid either false-positives or false-negatives. This may depend on the selected intervention.

For interventions that require a lot of time and resources, such as counseling at the student psychologist, false-positives need to be avoided. In this case, it may be more important to prevent wasting limited time and resources by using a screener with high specificity. A possible disadvantage is that many hazardous drinkers could be missed due to the lower sensitivity. This may, however, be acceptable because (1) hazardous drinking is not immediately life threatening and (2) for many students, heavy alcohol use and alcohol dependence in adolescence and early adulthood will tend to decline at older ages [38]. From our results, cutoff scores of 8 in females and 9 in males seem most suitable when screening students for interventions with high costs and resources.

The avoidance of false-negatives may be preferred for interventions with low cost and little personal effort. These interventions may take different forms, from merely providing information on the risks of hazardous drinking to personalized online advice and self-guided online interventions. Self-guided online interventions based on integrated therapeutic principles have been demonstrated to be effective in both community and health care settings and to be more effective than online interventions based on personalized normative feedback alone [39]. In this scenario, high sensitivity may be strived for. Although there will be more false-positives, providing some nonhazardous drinkers with advice and information to lower their alcohol consumption is not harmful. From our findings, a cutoff score of 7 in females and 8 in males may be most suitable when positively screened students are referred to an intervention with low costs and limited resources.

The health care cost for AUDs is high, and most interventions are cost-effective [40]. However, a low cutoff will result in more false-positives (i.e., identifying nonproblematic drinkers as problematic drinkers), who will increase the costs of the intervention, but not the effects. Moreover, false-positives may undermine the confidence of professionals in the screening instrument. Therefore, cost-effective interventions become ineffective when the threshold for referring individuals to the intervention is too low. Hence, for interventions that require a lot of time and resources, false-positives need to be avoided. For interventions with low costs and resources, the avoidance of false-positives is less necessary as the extra effects might be higher than the extra costs.

The AUDIT-C as an Indicator of Hazardous Alcohol Use

\section{Limitations}

This study has several limitations. First, the information on alcohol consumption is based on self-reports, which is generally found to be accurate, under specific conditions. Although studies showed that self-reported alcohol consumption levels and problems may stay underreported due to socially desirable answering of questions [41, 42], other studies showed that problematic drinkers' self-reports are generally valid across different cultures and ethnicities [43], especially when conducted in a research setting and participants were given assurances of confidentiality [44]. Collected data in the present study were processed anonymously, which was explicitly stated to the participants.

Second, participation in surveys might be selective. Not all students who were invited completed the survey. For the Student Health Check in Amsterdam, the total invited sample size is unknown, as there were multiple recruitment methods that could not be monitored. Only the response rate for Zwolle could be calculated. Although the respondents were similar in their basic characteristics (i.e., age, gender, academic year, faculty) compared to the general student population, the low response rate may affect reliability and validity of the study. In general, healthier people are more willing to cooperate in health research than unhealthy people [45]. This might lead to an underestimation of the proportion of hazardous drinkers and an underestimation of the alcohol consumption level. As a result, the PPV may be underestimated. Furthermore, the results are based on a Dutch sample, so it remains unclear to what extent they translate to other student samples, although we expect generalizability to countries with similar student cultures (e.g., many other European countries). Moreover, the data from the 2 different cohorts were collected in 2 different time periods, 2012/2013 and 2015/2016. However, we do not expect large differences in drinking behavior between these 2 periods, as the interval between them is small.

Third, the use of short scales has been advocated [46, 47]. Several studies discussed the challenges and caveats of short scales. Although they do not oppose the use of these scales, they do assert that the validity standards for short scales should be very high [46], in particular in clinical settings. It's important to strike a balance between maximizing the construct coverage (as in long scales) and the efficiency of measurement (as in short scales) [47]. According to Shrout and Yager [48], "it may be possible to use shortened versions of established screening scales for case identification and prevalence estimation without un-

Eur Addict Res 2020;26:1-9

DOI: $10.1159 / 000503342$ 
due cost in terms of sensitivity and specificity of the screen." This current study aimed to contribute to suggesting a specific cutoff for hazardous student drinking and makes a cautious step in that direction. Further research elaborating on this study and its proposed cutoffs is recommended.

Fourth, the gold standard for validating a screener is to use a validated clinical interview as outcome measure, which was not possible in our study.

Finally, future research may apply different statistical analyses that allow testing the extent to which the optimal AUDIT-C cutoff scores differ between subgroups and examine whether introducing more narrow subgroups that combine multiple risk factors for hazardous drinking leads to higher diagnostic validity.

\section{Conclusion}

Considering concurrent validity, the AUDIT-C performed well and has good potential as screener to identify hazardous drinking students at risk for AUD. The AUDIT-C also has clear advantages because of its brevity. A general cutoff score of 7 provided the most balanced combination of sensitivity and specificity for European students, or 7 (females) and 8 (males), when gender-specific cutoffs are used. We recommend that the AUDIT-C is primarily intended to determine students who are eligible for further alcohol assessments and, secondarily, targeted interventions. A cutoff higher or lower than 7 may be selected, when the importance of avoiding false-positives versus false-negatives needs to be considered in light of the preventive action that is undertaken with those identified as hazardous drinkers, at risk for AUD.

\section{Acknowledgments}

We would like to thank all students and employees of the University of Amsterdam and Windesheim University of Applied Sciences who made a contribution to this study and who supported the development of the web-based questionnaires.

\section{Statement of Ethics}

The Ethics Committee at the University of Amsterdam granted ethical approval for the Student Health Check project. The Executive Board of Windesheim University of Applied Sciences granted approval for the Study environment, Health, and Study Success survey. All subjects received written information about the aim of the study and participants participated voluntarily. The consent of the participants was obtained by virtue of survey completion. All data were analyzed anonymously. The authors have no ethical conflicts to disclose.

\section{Disclosure Statement}

The authors have no conflicts of interest to declare.

\section{Funding Sources}

This work was supported by the Netherlands Organization for Scientific Research (NWO) under grant number 023.004.118.

\section{Author Contributions}

All authors were responsible for the study design. J.M.D. and C.M.H. collected the data. S.V. and J.M.D. were responsible for the statistical analyses and interpretation of the data in agreement with all authors. S.V. and J.M.D. wrote the first version of the manuscript, and all authors participated in the revisions of the manuscript. All authors read and approved the final manuscript.

\section{References}

1 Dawson DA, Grant BF, Stinson FS, Chou PS. Another look at heavy episodic drinking and alcohol use disorders among college and noncollege youth. J Stud Alcohol. 2004 Jul;65(4): 477-88.

2 Kypri K, Cronin M, Wright CS. Do university students drink more hazardously than their non-student peers? Addiction. 2005 May; 100(5):713-4.

3 O'Malley PM, Johnston LD. Epidemiology of alcohol and other drug use among American college students. J Stud Alcohol Suppl. 2002 Mar;(14):23-39.

4 Stock C, Mikolajczyk R, Bloomfield K, Maxwell AE, Ozcebe H, Petkeviciene J, et al. Alco- hol consumption and attitudes towards banning alcohol sales on campus among European university students. Public Health. 2009 Feb;123(2):122-9.

5 Wicki M, Kuntsche E, Gmel G. Drinking at European universities? A review of students' alcohol use. Addict Behav. 2010 Nov;35(11): 913-24.

6 Wechsler H, Nelson TF. Binge drinking and the American college student: what's five drinks? Psychol Addict Behav. 2001 Dec; 15(4):287-91

7 Townshend JM, Duka T. Binge drinking, cognitive performance and mood in a population of young social drinkers.
Alcohol Clin Exp Res. 2005 Mar;29(3):31725.

8 Jennison KM. The short-term effects and unintended long-term consequences of binge drinking in college: a 10-year follow-up study. Am J Drug Alcohol Abuse. 2004 Aug;30(3): 659-84.

9 Bonnie, Richard J, O’Connell ME. Reducing underage drinking: a collective responsibility. National Academies Press; 2005.

10 Petit G, Maurage P, Kornreich C, Verbanck P, Campanella S. Binge drinking in adolescents: a review of neurophysiological and neuroimaging research. Alcohol Alcohol. 2014 MarApr;49(2):198-206. 
11 Karam E, Kypri K, Salamoun M. Alcohol use among college students: an international perspective. Curr Opin Psychiatry. 2007 May; 20(3):213-21.

12 The ESPAD group. ESPAD Report 2015. Results from the European School Survey Project on Alcohol and Other Drugs. European Monitoring Centre for Drugs and Drug Addiction. 2016.

13 Statline CB. Leefstijl en (preventief) gezondheidsonderzoek; persoonskenmerken. 2016. Den Haag/Heerlen. Available from: http:// statline.cbs.nl/StatWeb/publication/?DM=S LNL\&PA=83021ned.

14 van Dorsselaer S, Goossens FX. Alcohol-, tabaks- en drugsgebruik door studenten. 2015; 1-40. Available from: https://assets.trimbos. nl/docs/f5a4716f-a658-4a45-81ff-ac1682139 a4e.pdf.

15 National Institute on Alcohol Abuse and Alcoholism. College Drinking. 2014. Availablefrom: http://www.niaaa.nih.gov/alcohol-health/ special-populations-co-occuring-disorders/ college-drinking.

16 Misch DA. "Natural recovery" from alcohol abuse among college students. J Am Coll Health. 2007 Jan-Feb;55(4):215-8.

17 Vik PW, Cellucci T, Ivers H. Natural reduction of binge drinking among college students. Addict Behav. 2003 Jun;28(4):643-55.

18 Casswell S, Pledger M, Pratap S. Trajectories of drinking from 18 to 26 years: identification and prediction. Addiction. 2002 Nov;97(11) 1427-37.

19 Sher KJ, Grekin ER, Williams NA. The development of alcohol use disorders. Annu Rev Clin Psychol. 2005;1(1):493-523.

20 Toner P, Böhnke JR, McCambridge J. A systematic review of alcohol screening and assessment measures for young people: a study protocol. BMJ Open. 2017 Jun;7(5):e016406.

21 Babor TF, Higgins-Biddle JC, Saunders JB, Monteiro MG. The Alcohol Use Disorders Identification Test Guidelines for Use in Primary Care. World Health Organization; 2001.

22 Saunders JB, Aasland OG, Babor TF, de la Fuente JR, Grant M. Development of the Alcohol Use Disorders Identification Test (AUDIT): WHO Collaborative Project on Early Detection of Persons with Harmful Alcohol Consumption-II. Addiction. 1993;88(6): 791-804.

23 Bush K, Kivlahan DR, McDonell MB, Fihn $\mathrm{SD}$, Bradley KA. The AUDIT alcohol consumption questions (AUDIT-C): an effective brief screening test for problem drinking. Ambulatory Care Quality Improvement Project (ACQUIP). Alcohol Use Disorders Identification Test. Arch Intern Med. 1998 Sep; 158(16):1789-95

24 Barry AE, Chaney BH, Stellefson ML, Dodd $\mathrm{V}$. Evaluating the psychometric properties of the AUDIT-C among college students. J Subst Use. 2015;20(1):1-5.

25 Kelly TM, Donovan JE, Chung T, Bukstein OG, Cornelius JR. Brief screens for detecting alcohol use disorder among 18-20 year old young adults in emergency departments: Comparing AUDIT-C, CRAFFT, RAPS4-QF, FAST, RUFT-Cut, and DSM-IV 2-Item Scale. Addict Behav. 2009 Aug;34(8):668-74.

26 Demartini KS, Carey KB. Optimizing the use of the AUDIT for alcohol screening in college students. Psychol Assess. 2012 Dec;24(4): 954-63.

27 Cortés Tomás MT, Giménez Costa JA, Motos-Sellés P, Sancerni Beitia MD, Cadaveira Mahía F. The utility of the Alcohol Use Disorders Identification Test (AUDIT) for the analysis of binge drinking in university students. Psicothema. 2017 May;29(2):229-35.

28 Gezondheidsraad. Alcohol - Achtergronddocument bij Richtlijnen goede voeding 2015 Den Haag: Gezondheidsraad, 2015; publicatienr. A15/05.

29 Government of the Netherlands. Young people and alcohol. N.d. Available from: https:// www.government.nl/topics/alcohol/youngpeople-and-alcohol.

30 Van der Heijde CM, Vonk P, Meijman FJ. Self-regulation for the promotion of student health. Traffic lights: the development of a tailored web-based instrument providing immediate personalized feedback. Heal Psychol Behav Med. 2015.

31 Fleming MF, Barry KL, MacDonald R. The alcohol use disorders identification test (AUDIT) in a college sample. Int J Addict. 1991 Nov;26(11):1173-85.

32 Corp IB. Released 2016. IBM SPSS Statistics for Windows, Version 24.0. Armonk, NY: IBM Corp.

33 Hanley JA, McNeil BJ. The meaning and use of the area under a receiver operating characteristic (ROC) curve. Radiology. 1982 Apr; 143(1):29-36

34 Metz CE. Basic principles of ROC analysis Semin Nucl Med. 1978 Oct;8(4):283-98.

35 Campbell CE, Maisto SA. Validity of the AUDIT-C screen for at-risk drinking among students utilizing university primary care. J
Am Coll Health. 2018 Nov-Dec;66(8):77482.

36 Källmén H, Berman AH, Jayaram-Lindström N, Hammarberg A, Elgán TH. Psychometric Properties of the AUDIT, AUDIT-C, CRAFFT and ASSIST-Y among Swedish Adolescents. Eur Addict Res. 2019;25(2):68-77.

37 Reinert DF, Allen JP. The alcohol use disorders identification test: an update of research findings. Alcohol Clin Exp Res. 2007 Feb; 31(2):185-99.

38 Jackson KM, Sher KJ, Gotham HJ, Wood PK. Transitioning into and out of large-effect drinking in young adulthood. J Abnorm Psychol. 2001 Aug;110(3):378-91.

39 Riper H, Hoogendoorn A, Cuijpers P, Karyotaki E, Boumparis N, Mira A, et al. Effectiveness and treatment moderators of internet interventions for adult problem drinking: an individual patient data meta-analysis of 19 randomised controlled trials. PLoS Med. 2018 Dec;15(12):e1002714.

40 Cobiac L, Vos T, Doran C, Wallace A. Costeffectiveness of interventions to prevent alcohol-related disease and injury in Australia. Addiction. 2009 Oct;104(10):1646-55.

41 Krumpal I. Determinants of social desirability bias in sensitive surveys: A literature review. Quality \& Quantity: International Journal of Methodology. 2013;47(4):2025-47.

42 Ogan C, Karakuş T, Kurşun E. Methodological Issues in a Survey of Children's Online Risk-Taking and Other Behaviours in Europe. J Child Media. 2013;7:1, 133-50.

43 Sznitman SR, Bord S, Elias W, Gesser-Edelsburg A, Shiftan Y, Baron-Epel O. Cross-Cultural Validity in Self-Reported Alcohol Use. Eur Addict Res. 2017;23(2):71-6.

44 Sobell LC, Sobell MB. Self-report issues in alcohol abuse: state of the art and future directions. Behav Assess. 1990;12(1):77-90.

45 Knudsen AK, Hotopf M, Skogen JC, Øverland S, Mykletun A. The health status of nonparticipants in a population-based health study: the Hordaland Health Study. Am J Epidemiol. 2010 Dec;172(11):1306-14.

46 Ziegler M, Kemper CJ, Kruyen P. Short Scales - Five Misunderstandings and Ways to Overcome Them. J Individ Differ. 2014;35(4):1859.

47 Smith GT, McCarthy DM, Anderson KG. On the sins of short-form development. Psychol Assess. 2000 Mar;12(1):102-11.

48 Shrout PE, Yager TJ. Reliability and validity of screening scales: effect of reducing scale length. J Clin Epidemiol. 1989;42(1):69-78.
The AUDIT-C as an Indicator of Hazardous Alcohol Use
Eur Addict Res 2020;26:1-9

DOI: $10.1159 / 000503342$ 